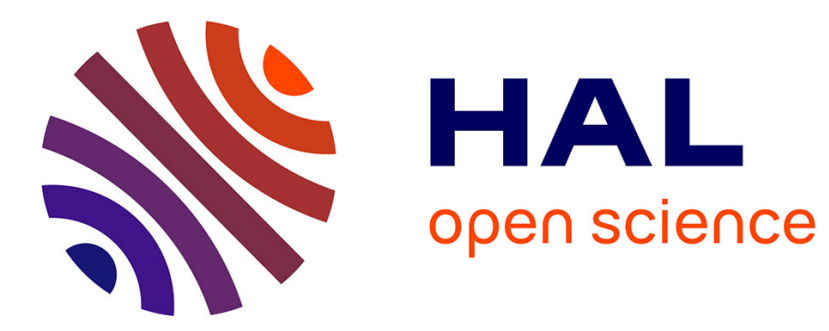

\title{
A differential geometry model for the perceived colors space
}

Edoardo Provenzi

\section{To cite this version:}

Edoardo Provenzi. A differential geometry model for the perceived colors space. International Journal of Geometric Methods in Modern Physics, 2016, 10.1142/S0219887816300087 . hal-01339467

\section{HAL Id: hal-01339467 \\ https://hal.science/hal-01339467}

Submitted on 29 Jun 2016

HAL is a multi-disciplinary open access archive for the deposit and dissemination of scientific research documents, whether they are published or not. The documents may come from teaching and research institutions in France or abroad, or from public or private research centers.
L'archive ouverte pluridisciplinaire HAL, est destinée au dépôt et à la diffusion de documents scientifiques de niveau recherche, publiés ou non, émanant des établissements d'enseignement et de recherche français ou étrangers, des laboratoires publics ou privés. 


\title{
A differential geometry model for the perceived colors space
}

\author{
Edoardo Provenzi* \\ Laboratoire MAP5 (UMR CNRS 8145), \\ Université Paris Descartes, 75006, Paris, France
}

\begin{abstract}
The space of perceived colors, before acquiring an industrial interest, has received a systematic theoretical attention from philosophers, physicists and mathematicians. The research about this topic is still active nowadays. In this paper it will be presented a critical overview of a model based of differential geometry proposed by H.L. Resnikoff in 1974. It will be shown that, while some fundamental and elegant ideas behind this model can be still used as a guiding principle, some other parts of the model must be updated to comply with the modern findings about color perception.
\end{abstract}

\section{Introduction}

The study of color has fascinated some of the most brilliant minds throughout the history. Among other we can indeed quote: Plato and Aristoteles in ancient Greece; Descartes, Hooke and Newton in the seventeenth century; Euler, Dalton, Young, Helmholtz, Grassmann, Hering, Riemann and Maxwell between the eighteen and the nineteen century and, very noticeably, Schrödinger in the twentieth century. The list is much longer if we consider poets and philosophers as Goethe, Locke, Schopenhauer and Wittgenstein.

What we call color is a sensation generated by the human brain in response to the interaction with a visual scene. Thus, color is a complex phenomenon in which account physics, neurophysiology and cognitive psychology converge.

In this paper we deal with the so-called space of perceived colors, a mathematical structure which serves a basic framework for the study of color image processing. In particular, we will consider a model for the space of perceived colors proposed by H.L. Resnikoff in 1974 in the paper [1], which is based on differential geometry and Lie group theory.

*edoardo.provenzi@parisdescartes.fr 
Resnikoff's model is founded on Schrödinger's axioms of color vision [2], to which he added a further axiom of homogeneity. He then used Lie groups and algebras representation theory to infer the metric structure of the color space. He has shown that the presence of the homogeneity axiom implies that the choice of the metric structure of the space can be only two-fold: the first metric agrees with that proposed by Stiles starting from colorimetric considerations, while the second is a novel metric has not been tested yet in color theory. A clever analysis of these two metric models in terms of Jordan algebras, which serves as a unifying background, completed his analysis of the color space.

In spite of its elegance and innovative character, Resnikoff's work did not raised the interest it deserved and fell into oblivion. This contribution has the aim to revive Resnikoff's ideas and to embed his model into a more modern conception of color, in particular the 'color in context' theory in the spatial and frequency domain, see e.g. $[6,7,8,9,10]$. This will be done trough a critical revision of some of the hypotheses used in his theory.

\section{Schrödinger's axioms on the space of perceived lights}

The Human Visual System, HVS from now on, can be roughly characterized as the union of the eyes and the part of the brain dedicated to the analysis of visual signals, i.e. rays of light $x(\lambda)$ coming from a visual scene with a wavelength $\lambda \in \Lambda$, where $\Lambda=[380,780] \mathrm{nm}$ is the spectrum of visible wavelengths.

In Resnikoff's model the HVS is interpreted as a black box with certain phenomenological features that will be discussed later and no intrinsic neurophysiological property is taken into account.

He considers a visible light $x: \Lambda \rightarrow \mathbb{R}^{+}$as an element of $L^{2}(\Lambda)$ and then he introduces an equivalence relation on $L^{2}(\Lambda)$ derived from the phenomenon of metamerism.

We say that two superpositions of visible lights $x$ and $y$ are metameric, and we write $x \sim y$, if they produce the same sensation to a human observer ${ }^{1}$. We write with $[x]$ the metameric class of $x$, i.e. the set of all spectral combinations of visible lights which give rise to the same perceived light. The space of perceived lights is defined as the quotient space

$$
\mathcal{P}=L^{2}(\Lambda) / \sim
$$

Schrödinger's axioms [2] combined a series of observations on the structure of $\mathcal{P}$ in an organic way. The axioms are as follows:

- Axiom 1 (Newton 1704 [3]): if $x \in \mathcal{P}$ and $\alpha \in \mathbb{R}^{+}$, then $\alpha x \in \mathcal{P}$;

- Axiom 2: if $x \in \mathcal{P}$ then it does not exist any $y \in \mathcal{P}$ such that $x+y=0$;

\footnotetext{
${ }^{1}$ With human observer here we mean an average observer with no visual deficiencies.
} 
- Axiom 3 (Grassmann 1853 [4], Helmholtz 1867 [5]): for every $x, y \in \mathcal{P}$ and for every $\alpha \in[0,1], \alpha x+(1-\alpha) y \in \mathcal{P}$;

- Axiom 4 (Grassmann $1853[4]): \forall\left\{x_{k}, k=1, \ldots, 4\right\} \subset \mathcal{P}, \exists \alpha_{k} \in \mathbb{R}$ such that $\sum_{k=1}^{4} \alpha_{k} x_{k}=0$.

Every axiom is the mathematical translation of physiological observations on color perception. Axiom 1 is easily interpreted: it simply means that $\mathcal{P}$ is a cone in $V$.

Axiom 2 mathematically means that $\mathcal{P}$ does not have one dimensional subspaces and it is the translation of the fact that no superposition of visible lights is metameric equivalent to the absence of light.

Axiom 3 is the most important for the later mathematical development, it implies that $\mathcal{P}$ is convex: i.e. every two colors in $\mathcal{P}$ can be joined by a line segment.

Axiom 4 states that every collection of more than three perceived colors is a linearly dependent family in the vector space $V$ spanned by the elements of $\mathcal{P}$, i.e. $\operatorname{dim}(V) \leq 3$ : when the dimension is 3 we talk about trichromatic vision. When $\operatorname{dim}(V)=2$ we refer to dichromatic vision, $\operatorname{dim}(V)=1$ is the case of monochromatic vision and $\operatorname{dim}(V)=0$ means blindness.

\section{Resnikoff's axiom of homogeneity and its con- sequences}

The main idea of Resnikoff was to add a further axiom, precisely the axiom of 'local homogeneity' of $\mathcal{P}$ with respect to changes of background illumination of the visual scene. Let us remember that if $X$ is a topological space and $G$ is a group of transformations acting on it, then $X$ is a homogeneous space with respect to $G$ if, for any two points $x, y \in X$, there exists a transformation $g \in G$ such that $g(x)=y$, i.e. any two points of $X$ can be joined by a suitable transformation $g$ induced by $G$.

$X$ is locally homogeneous with respect to $G$ if this property holds only locally, i.e. for every $x \in X$ there is an open neighborhood $U_{x}$ containing it and such that every $x^{\prime} \in U_{x}$ can be written as $x^{\prime}=g(x)$ for a certain $g \in G$.

The reason why Resnikoff is lead to consider these mathematical structures is that any perceived light $x \in \mathcal{P}$ can be transformed in a perceived light $y \in \mathcal{P}$ not too different from $x$ by a change of background illumination, and this process is reversible.

Resnikoff claims that this group can be assumed as the following:

$$
G L(\mathcal{P}):=\{g \in G L(V) \mid g(x) \in \mathcal{P} \quad \forall x \in \mathcal{P}\},
$$

where $G L(V)$ is the group of orientation-preserving invertible linear operators on $V$, or, equivalently, the group of real $n \times n$ matrices with positive determinant, where $n=\operatorname{dim}(V) \leq 3$ thanks to Axiom 4 . 
He justifies this choice from the consideration that Axiom 1 implies that $\mathcal{P}$ is a cone embedded in $V$ and so a general transformation of illumination must preserve the orientation of the cone and it must also be invertible, since it is possible to turn back to the initial conditions of illuminations. The condition $g(x) \in \mathcal{P}$ is motivated by the fact that, after the change of illumination, we can still perceive colors.

Now, if we accept Axiom 3, we have that for every couple of perceived colors $x, y \in \mathcal{P}$ there exists the line segment that join $x$ to $y$. This segment is compact, hence it can be covered by a finite partition of open neighborhoods $U_{1}, \ldots, U_{n}$ and the color $x$ can be moved along this line segment passing from a neighborhood to the next one with the transformations $g_{1}, \ldots, g_{n}$.

Obviously the (global) transformation that enables to pass from $x$ to $y$ is the composition of the single transformations, i.e. $y=g(x), g=g_{n} \circ \cdots \circ g_{1}$.

Thus, if the space of perceived colors is assumed to be convex, then local homogeneity is equivalent to global homogeneity and, for this reason, Resnikoff is lead to postulate his fifth axiom on the structure of the color space as follows:

- Axiom 5 (Resnikoff 1974): $\mathcal{P}$ is globally homogeneous with respect to the group of transformations of illumination $G L(\mathcal{P})$.

The significance of this axiom relies on the fact that homogeneous spaces have useful properties that Resnikoff used to determine the structure of $\mathcal{P}$ explicitly.

In fact it is known that if $K$ denotes the isotropy subgroup ${ }^{2}$ of $G L(\mathcal{P})$ in any point $x \in \mathcal{P}$, then

$$
\mathcal{P} \simeq G L(\mathcal{P}) / K \text {. }
$$

Using results from Lie groups and algebras theory, Resnikoff proved that only two kind of spaces are compatible with the homogeneous structure of $\mathcal{P}$ :

$$
\mathcal{P} \simeq \mathbb{R}^{+} \times \mathbb{R}^{+} \times \mathbb{R}^{+} \quad \text { or } \quad \mathcal{P} \simeq \mathbb{R}^{+} \times S L(2, \mathbb{R}) / S O(2) .
$$

The first space agrees with the so-called Helmholtz-Stiles trichromatic space, where each non-negative coordinate can be put in relationship with the excitation of the retinal cones responding to the Low, Middle and Short wavelengths, typically called LMS coordinates.

The three LMS coordinates are then linearly transformed to fulfill different purposes, giving birth to a class of three-dimensional spaces to which belong the so-called trichromatic spaces RGB, XYZ, and so on, see e.g. [12].

The second geometric structure instead is novel: $\mathbb{R}^{+}$can be interpreted as an achromatic coordinate, but no clear explanation of the Poicaré-Lobachevsky two-dimensional space of constant negative curvature $S L(2, \mathbb{R}) / S O(2)$ in terms of color features is available yet.

\footnotetext{
${ }^{2}$ In general, if $X$ is a homogeneous space w.r.t. $G$, then the isotropy subgroup $G_{x}$ of $G$ in a point $x \in X$ is the subgroup of transformations of $G$ that leaves $x$ fixed, i.e. $G_{x}:=\{g \in$ $G \mid g(x)=x\}$. All the isotropy subgroups, as $x$ varies in $X$, are conjugated to each other, i.e. $G_{y}=\tilde{g} G_{x} \tilde{g}^{-1}, y \in X, \tilde{g} \in G$.
} 
Once determined the only two possible structures of $\mathcal{P}$, Resnikoff used a phenomenological property of human vision, namely color constancy, as an invariance principle which allowed him determining the metric on $\mathcal{P}$. In the colorimetric interpretation, a metric on $\mathcal{P}$ allows measuring perceptual differences of color in a visual scene.

Color constancy is the name reserved to the robustness of perception of color differences with respect to global illumination changes. If we indicate with $d$ the metric which measures the difference of colors in $\mathcal{P}$, then color constancy implies that $d$ must be $G L(\mathcal{P})$-invariant, i.e.

$$
d(x, y)=d(g(x), g(y)) \quad \forall x, y \in \mathcal{P}, \quad \forall g \in G L(\mathcal{P}) .
$$

Resnikoff showed that there is only one metric compatible with this invariance for both spaces represented in (4). If $\mathcal{P} \simeq \mathbb{R}^{+} \times \mathbb{R}^{+} \times \mathbb{R}^{+}$, then

$$
d s^{2}=\alpha_{1}\left(\frac{d x_{1}}{x_{1}}\right)^{2}+\alpha_{2}\left(\frac{d x_{2}}{x_{2}}\right)^{2}+\alpha_{3}\left(\frac{d x_{3}}{x_{3}}\right)^{2},
$$

where $x_{j} \in \mathbb{R}^{+}$and $\alpha_{j}$ are positive real constants, for $j=1,2,3$. This metric agrees with the Helmholtz-Stiles color distance.

If $\mathcal{P} \simeq \mathbb{R}^{+} \times S L(2, \mathbb{R}) / S O(2)$, then one must use the following parameterization $\mathcal{P} \ni x=\left(\begin{array}{ll}x_{1} & x_{3} \\ x_{3} & x_{2}\end{array}\right), x$ is a $2 \times 2$ positive-definite real symmetric matrix, $x=\operatorname{det}(x)\left(\frac{x}{\operatorname{det}(x)}\right), \operatorname{det}(x) \in \mathbb{R}^{+}$and $\frac{x}{\operatorname{det}(x)} \in S L(2, \mathbb{R}) / S O(2)$. With this notation we have:

$$
d s^{2}=\operatorname{Tr}\left(x^{-1} d x x^{-1} d x\right),
$$

where $\operatorname{Tr}$ is the trace operator, which guarantees invariance thanks to its cyclic property. Again, this is a novel color metric that has never been studied.

Once we have color metrics on $\mathcal{P}$, the perceptual difference among two colors $x, y \in \mathcal{P}$ can be calculated with the integral

$$
d(x, y)=\int_{\gamma} d s \quad \gamma(0)=x, \gamma(1)=y,
$$

where $\gamma$ is the geodesic which connects $x$ to $y$.

In particular, Resnikoff computed $d(x, y)$ in the case $\mathcal{P} \simeq \mathbb{R}^{+} \times \mathbb{R}^{+} \times \mathbb{R}^{+}$ and $y_{j}=\lambda x_{j}, \lambda \in \mathbb{R}^{+}$, i.e. when $x$ and $y$ differ only in their intensities. In this situation the metric will measure the brightness difference. The calculation of the integral gives

$$
d(x, \lambda x)=\sqrt{\alpha_{1}^{2}+\alpha_{2}^{2}+\alpha_{3}^{2}} \int_{1}^{\lambda} \frac{d t}{t}=\sqrt{\alpha_{1}^{2}+\alpha_{2}^{2}+\alpha_{3}^{2}} \log (\lambda),
$$

i.e. the difference in brightness is proportional to the logarithm of the intensity difference. This is coherent with what stated by Weber-Fechner's law [11]. 
To conclude, it has to be quoted that Resnikoff used the formalism of Jordan algebras ${ }^{3}$ to provide a compact coherent framework where both representations of $\mathcal{P}$ can be treated in a uniform way.

Within the Jordan algebras framework he studied surfaces of constant brightness, how to define hue and saturation of a perceived light, complementary colors and how to extend the previous results to the presence of multiple lights.

\section{Critics to some assumptions of Resnikoff's model and perspectives for future research}

Resnikoff's model remains one of the most elegant treatises on color perception and the little attention that it has received from the colorimetric community is not justified. The principal aim of this paper is to honor Resnikoff's work, which has paved the road to the introduction of some advanced mathematical techniques typically used in theoretical physics, e.g. differential geometry, Lie groups and algebras representation theory and Jordan algebras, to the theory of color perception.

In this final section, we would also like to underline some oversimplified assumptions and omissions which are present in Resnikoff's model with the hope that this consideration could raise the interest for an improvement of the geometrical theory of the space of perceived colors.

First of all, Axiom 1 fails for $\alpha \simeq 0$ and $\alpha \gg 1$. In fact, as $\alpha$ approaches zero, the retinal cones responsible for color vision do not work anymore and retinal rods are activates, allowing only black and white vision, which can be identified with achromatic colors in $\mathcal{P}$. However, rods sensitivity is finite, so that under a certain threshold $\bar{\alpha}$, vision ceases and with it the geometric structure of $\mathcal{P}$. The same can be said when $\alpha$ overcomes an upper limit, after with retinal cones saturate and sight is lost.

Then, in Resnikoff's model only independent light stimuli over a uniform background are considered, however color vision in real world conditions is much more complex. In fact, color perception of natural scenes is intrinsically local: as Figure 1 shows, the hue, saturation and brightness of a patch strongly depend on the surrounding patches, a phenomenon called 'induction'. This is the reason why one must distinguish between spectral colors of light sources isolated from the rest of the visual field, and color in context.

Induction analysis is an active research field both in image processing and cognitive psychology. In particular, in [13] and [14] the authors proposed a computational method to measure achromatic brightness induction taking inspiration from one of the most famous models of color perception: the Retinex theory $[6,7]$.

When induction phenomena are taken into account, it is clear that if we

\footnotetext{
${ }^{3} \mathrm{~A}$ Jordan algebra is a (nonassociative) algebra over a field whose multiplication is commutative and satisfies Jordan's identity: $(x y)(x x)=x(y(x x))$. They are used, among others, to provide a formalization of the notion of algebra of observables in quantum mechanics.
} 
want to represent color differences a spatially variant Riemannian metric on $\mathcal{P}$ must be considered, instead of a global one.

Finally, the global homogeneous hypothesis, which lies at the basis of Resnikoff's theory, may hold for isolated lights on a uniform background, but in a complex scene with enough contrasts human color constancy is activated and visual perception becomes very robust with respect to illumination changes.

Other non-uniformities, as those singled out by McAdams in [15], must be also taken into account.

All these observations show that, if we want to model real world color vision in natural scenes the set of Schrödinger plus Resnikoff axioms must be suitably

modified. The analysis of this new paradigm opens the possibility to introduce in colorimetry even more sophisticated mathematical techniques inspired from theoretical and mathematical physics.

\section{References}

[1] H.L. Resnikoff, Differential geometry and color perception, Journal of Mathematical Biology 1 (1974), 97-131.

[2] E. Schrödinger, Grundlinien einer Theorie der Farbenmetrik im Tagessehen (Outline of a theory of colour measurement for daylight vision), Annalen der Physik 63 (4) (1920), 397-456; 481-520. Available in English in Sources of Colour Science, Ed. David L. MacAdam, The MIT Press (1970), 13482.

[3] I. Newton, Opticks, London, Smith and Walford (1704).

[4] H.G. Grassmann, Zur Theorie der Farbmischung, Poggendorff's Annalen der Physik, (89) (1853), 69-84.

[5] H. von Helmholtz, Handbuch der physiologischen Optik, Allgemeine Encyclopdie der Physik, IX. Band. Leipzig, Leopold Voss (1867).

[6] E.H. Land, J.J. McCann, Lightness and Retinex Theory, Journal of the Optical Society of America, 61 (1) (1971), 1-11.

[7] E. Provenzi, L. De Carli, A. Rizzi, D. Marini, Mathematical definition and analysis of the Retinex algorithm, Journal of the Optical Society of America A, 22 (12) (2005), 2613-2621.

[8] R. Palma-Amestoy, E. Provenzi, M. Bertalmío, V. Caselles, A perceptually inspired variational framework for color enhancement, IEEE Transactions on Pattern Analysis and Machine Intelligence, 31 (3), (2009), 458-474.

[9] E. Provenzi, V. Caselles, A Wavelet Perspective on Variational PerceptuallyInspired Color Enhancement, International Journal of Computer Vision, 106 (2), 153-171.

[10] M.D. Fairchild, Color appearance models, Wiley, (2005). 
[11] G. Wyszecky and W.S. Stiles, Color science: Concepts and methods, quantitative data and formulas, Wiley $\&$ Sons (1982).

[12] T. Gevers, A. Gijsenij, J. van de Weijer, J-M. Geusebroek, Color in Computer Vision, Fundamentals and Applications, Wiley, (2012).

[13] H. Wallach, H., Brightness constancy and the nature of achromatic colors, Journal of Experimental Psychology, 38 (3) (1948), 310-324.

[14] M.E. Rudd, I.K. Zemach, Quantitive properties of achromatic color induction: An edge integration analysis, Vision Research, 44 (2004), 971-981.

[15] D.L. MacAdams, Visual sensitivities to colour differences in daylight, Journal of the Optical Society of America A, 32 (5), (1942), 247-274. 


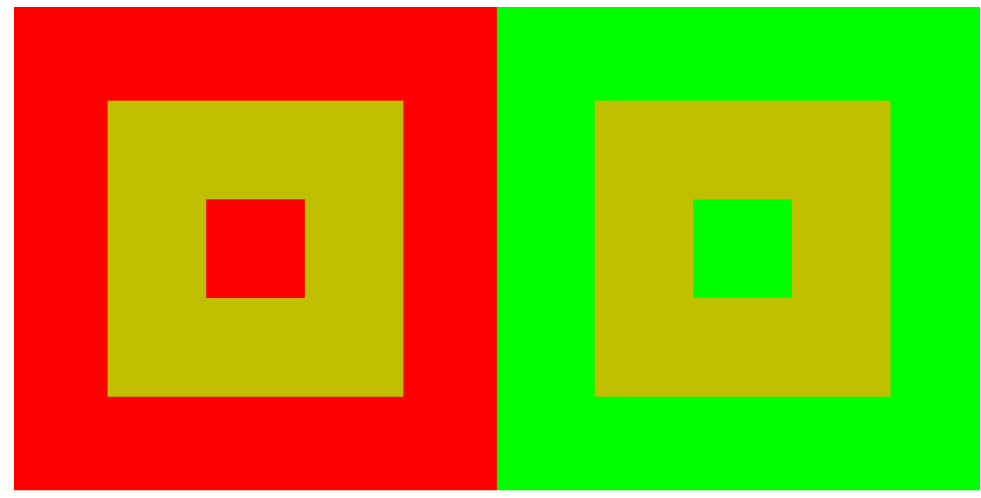

\section{Induced Hue}

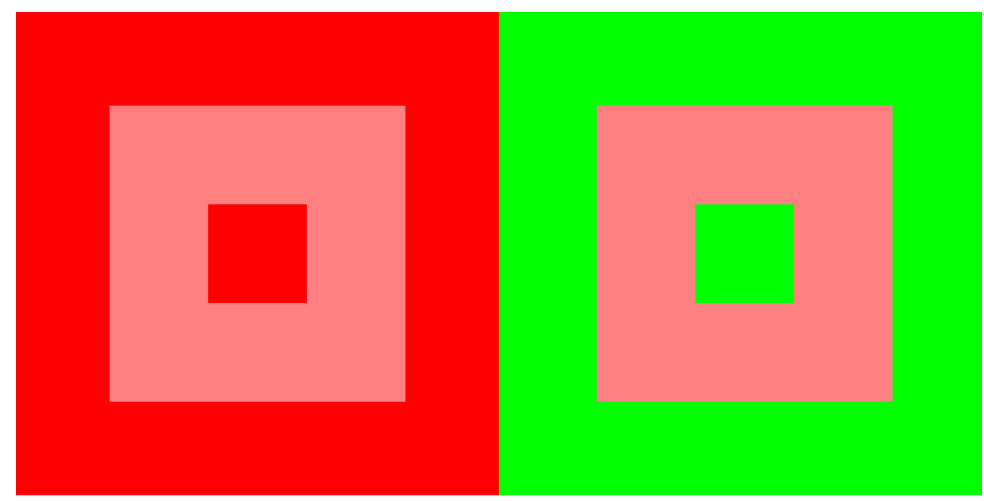

Induced Saturation

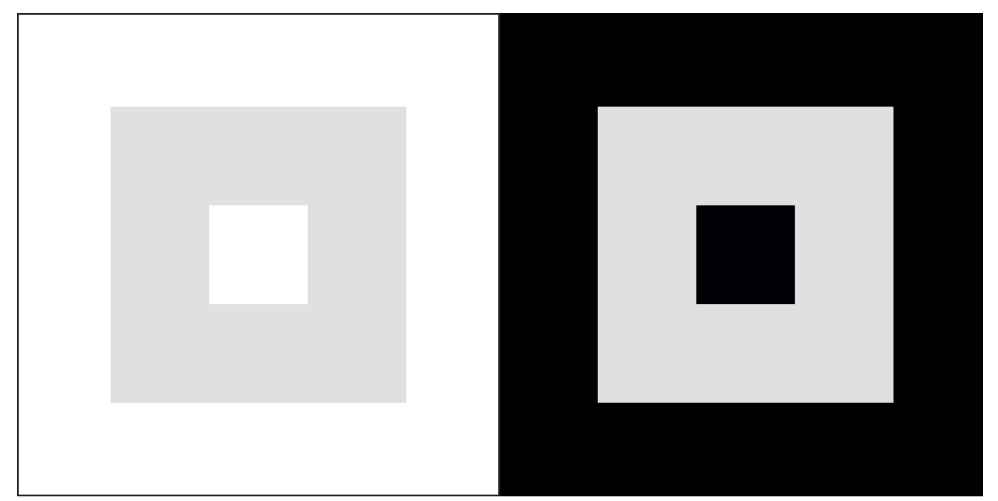

\section{Induced Brightness}

Figure 1: Top to bottom: the induction phenomena. In all the pictures, the pierced square is the same in both columns, however it is surrounded, inside and outside, by another stimulus, which is different in the two columns. The top row shows that the (fixed) hue (tint) of the the pierced square is perceived as greenish or reddish depending on the surrounding. The middle row shows that the (fixed) saturation (color purity) of the the pierced square is perceived more or less intense with respect to the neighborhoods. Finally, in the bottom row, we can see that the brightness (perceived luminosity) of the pierced square changes dramatically when the surrounding area changes. 